\title{
Design for Additive Manufacturing Based on Axiomatic Design Method
}

\author{
Konstantinos Salonitis \\ Manufacturing Department, Cranfield University, Bedford, UK \\ k.salonitis@cranfield.ac.uk
}

\begin{abstract}
Additive manufacturing technology promises to revolutionize the way products are manufactured and supplied to the customer. Existing design methods however do not take full advantage of the additive manufacturing processes capabilities. This paper presents a framework to improve the current design approach for additive manufacturing using an axiomatic design approach. The proposed framework is used both for the development of new products and the re-designing of existing products that are designed for conventional manufacturing. A case study is presented for the validation of the framework that highlights how this method can be used for design validation and decision making.
\end{abstract}

Keywords: Product development; design method; additive manufacturing 


\section{Introduction}

Conventional manufacturing processes, such as machining, pose limitations on the component geometries that can be produced. These limitations often result in structures that are inefficient, as many areas of a component have excess material that cannot be removed physically or in a cost effective way through conventional methods. Additive Manufacturing (AM) processes provide the opportunity to address the problem of inefficient structures. They allow components to be manufactured in a bottom-up approach with laying material only where it is required. One of the key advantages of such processes is the fabrication of components and even complete assemblies directly derived from a 3D CAD model without the need for process planning in advance of manufacturing. Various methods that allow the "building" of three-dimensional objects in sequence by adding layers over each other have been developed [1].

AM technology has a relative short history of about 25 years and it has grown largely since its invention: according to Wohlers Report [2], the AM projected value for 2015 is $\$ 4 \mathrm{bn}$, and will reach \$6bn in 2017 and almost \$11bn in 2021. However, although it is becoming more and more mature, often claimed as the "next industrial revolution", there are still a number of challenges for the successful commercialisation. AM technology challenges are related to the materials, the available CAD software, the data management, the sustainability, the affordability, the process speed, the process reliability, the intellectual property, and the standards to name few [3]. The design for AM has been also identified as a key challenge, highlighting that for exploiting the capabilities that AM processes offer, the designers have to adapt their approach to the AM technology, not replicating the existing methods and philosophies established for conventional processes.

AM processes can be classified into three different categories depending on the status of the material used to create the part during the process such as powder based, liquid based and solid based (Figure 1). A large number of different AM processes have been developed in the short history of AM; few of them though survived over time. Common materials are aluminium, steel alloys, precious metals, plastics used in a powder form and paper; but wood, wax, paper, clay, concrete, sugar and chocolate are possible to be used as filament. Selective laser sintering (SLS), electron beam melting (EBM), laser powder forming (LPF), binder jetting (BJ) are applicable for metals, for prototype and direct part manufacturing 
purposes. LPF is applicable for repair of parts and can thus extend the lifetime of a product even further. BJ's ability to produce complex sand casting moulds has the potential of design optimisation, where less material would be used in the mould. Ultrasonic additive manufacturing (UAM) and laminated object manufacturing (LOM) are suitable for metal artefacts, whereas LOM is additionally considered suited for paper and plastic artefacts. UAM's ability for interchangeable metals during the layering process offers opportunities for the production and repair of metal material of more than one type, such as bimetals where different coefficient of thermal expansion are required. Fused deposition modelling (FDM) with polymer based material and using stereolithography (SL) and digital light processing (DLP) with photopolymer based material are used mainly for prototypes manufacturing.

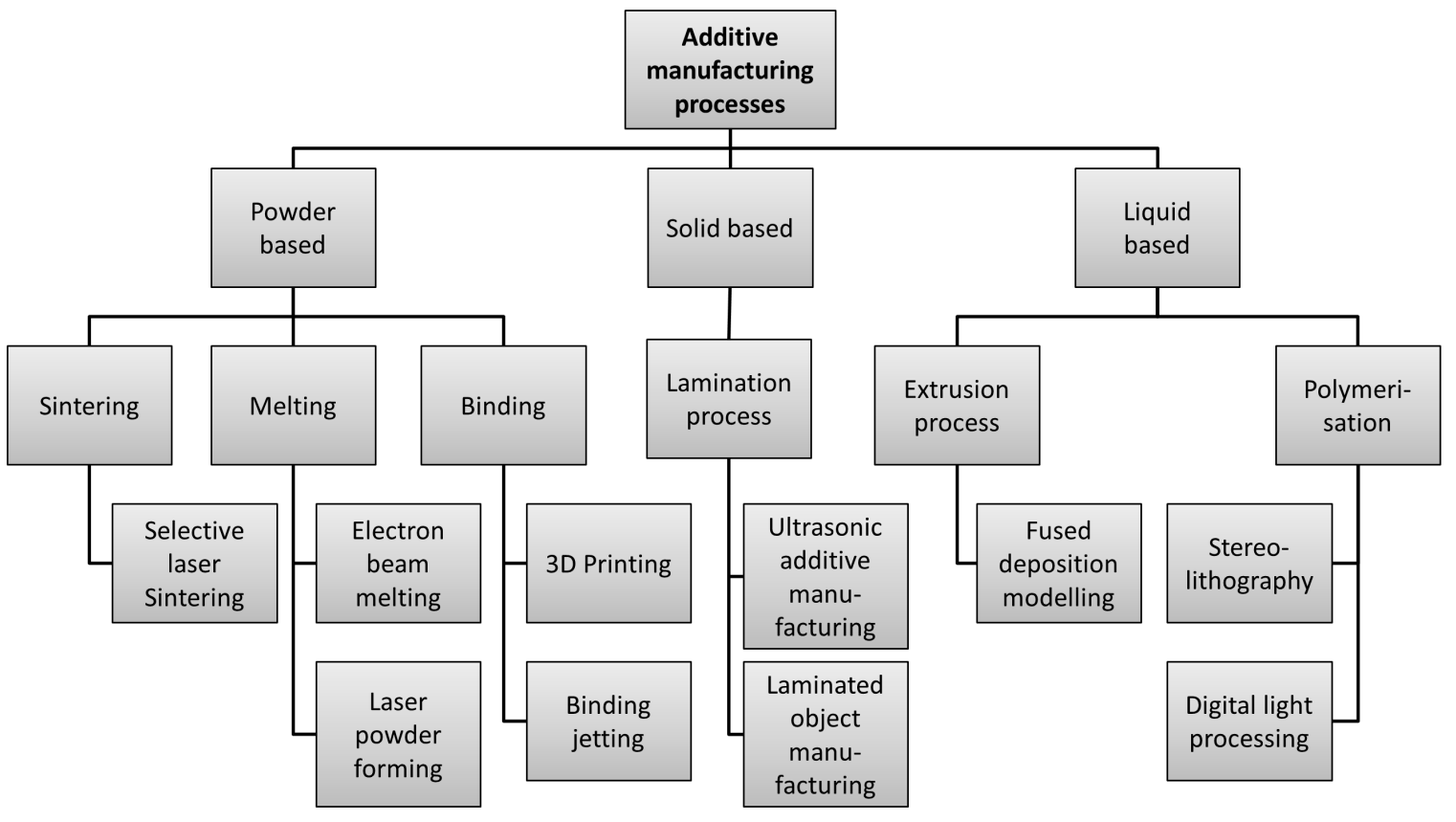

Figure 1. Additive manufacturing processes classification (updated from Kruth et al. [1])

The 3D model of a product is traditionally generated via computer-aided design (CAD). Material is added layer by layer, derived as thin cross-section from the 3D model. The layer thickness determines the resolution of the manufactured product.

Components optimised to exploit the benefits provided by additive manufacturing techniques can look very different from those designed to suit conventional production methods. It is however challenging for engineers accustomed to designing components for 
conventional manufacturing to adapt their thinking to exploit the additive manufacturing capabilities.

\section{Design for additive manufacturing}

AM technologies allow for the creation of models and products that are intricate in nature and made of composite materials which can be customised. Such processes can be defined as the ones in which physical objects are made through layer by layer selective fusion, polymerisation or sintering of materials, depending on the underline principle of the process [4]. After the design has been finalized, the designer has to follow a number of steps (such as slicing, support generation etc.) that are required for the additive manufacturing of a part; these steps may vary with the technology used.

Since additive methods remove most of the limitations of conventional manufacturing, any complex design can be directly transformed into the final product. Conventional manufacturing design constraints, such as avoidance of sharp corners, minimising weld lines, draft angles and constant wall thickness are obsolete in that case. This allows designers to closely adhere to the initial concept design and specification.

Design methodologies that have been developed for manufacturing are attempting to constrain designer's imagination based on the manufacturing processes capabilities. For example limitations due to the use of tooling are not relevant to additive manufacturing processes. For the conventional processes, a number of design methodologies have been presented such as design for manufacturing and design for assembly with a number of variations for specific processes and industrial sectors.

However, with regards to the design frameworks for using AM processes, few studies have been published. Indicatively Rodrigue and Rivette [5] developed a design methodology based on design for assembly notion, borrowing ideas from TRIZ analysis, for the optimization of the alternative designs. Vayre et al. [6] presented a methodology composed of four steps. Podshivalov et al. [7] focused on the design for additive manufacturing in medical applications. Ponche et al. [8] took into consideration the part orientation during building, the functional optimization and the optimization of the manufacturing paths. Adam and Zimmer [9] documented a number of design rules for additive manufacturing that 
can be integrated in a design framework. Salonitis and Saeed [10] presented a decision support method for the redesign of existing products using additive manufacturing.

A common characteristic of all the studies reviewed is that the additive manufacturing capabilities are not considered early enough on the design phase. Among the different design theories and methodologies, axiomatic design theory considers and assesses good design ideas even from the concept design phase, and thus looks as a promising approach.

Axiomatic design [11], [12] was introduced in an attempt to scientifically define the design process. Since its introduction numerous papers have been presented applying the method for the development of new products none though on the design for AM. Recently a thorough literature review was presented indicating that most of the relevant studies are application based using mostly the independence axiom [13].

The objective of the present paper is to investigate the idea of using axiomatic design method for the conceptual design of a component to be manufactured using additive manufacturing.

\section{Proposed framework}

Axiomatic design is based on mapping the customer needs on functions that the object is expected to perform (defined as functional requirements - FRs), then derive design parameters (DPs) indicating how the object can satisfy such FRs and finally describe the process variables (PVs) for the manufacturing of the object. This process is usually implemented through zigzag decomposition having in mind two fundamental design axioms, the independence axiom (each functional requirement should be independent) and the information axiom (select the design alternative with the minimum information content).

The method is ideal for developing new product designs and assessing the designs early in the process. However, the manufacturing process constraints and capabilities are not considered directly during the transition from the functional to the physical domain. The mapping is focused on two adjacent domains in order to interlink "what we want" and "how to achieve what we want" [12]. Axiomatic design thus considers the manufacturing of the component after the design has been defined in the physical domain and it is described in the process domain through the process variables. 
Nevertheless, a number of theorems and corollaries that have been presented by Suh [12] consider the manufacturability of a product. For example the third corollary suggests the integration of physical parts, with additive manufacturing providing large capabilities in such design approach. Suh discussed in detail how axiomatic design can be used for assisting manufacturing [14].

The proposed approach for taking into consideration the manufacturing capabilities and limitations is depicted in Figure 2. The core of the proposed framework is the axiomatic design decomposition of the design space into domains (shown as ellipses in Figure 2), however in order for the manufacturability of the design to be improved from the early design phases, in addition to the theorems and corollaries, information such as manufacturing guidelines need to be fed into the functional and physical domain during the decomposition of these domains.

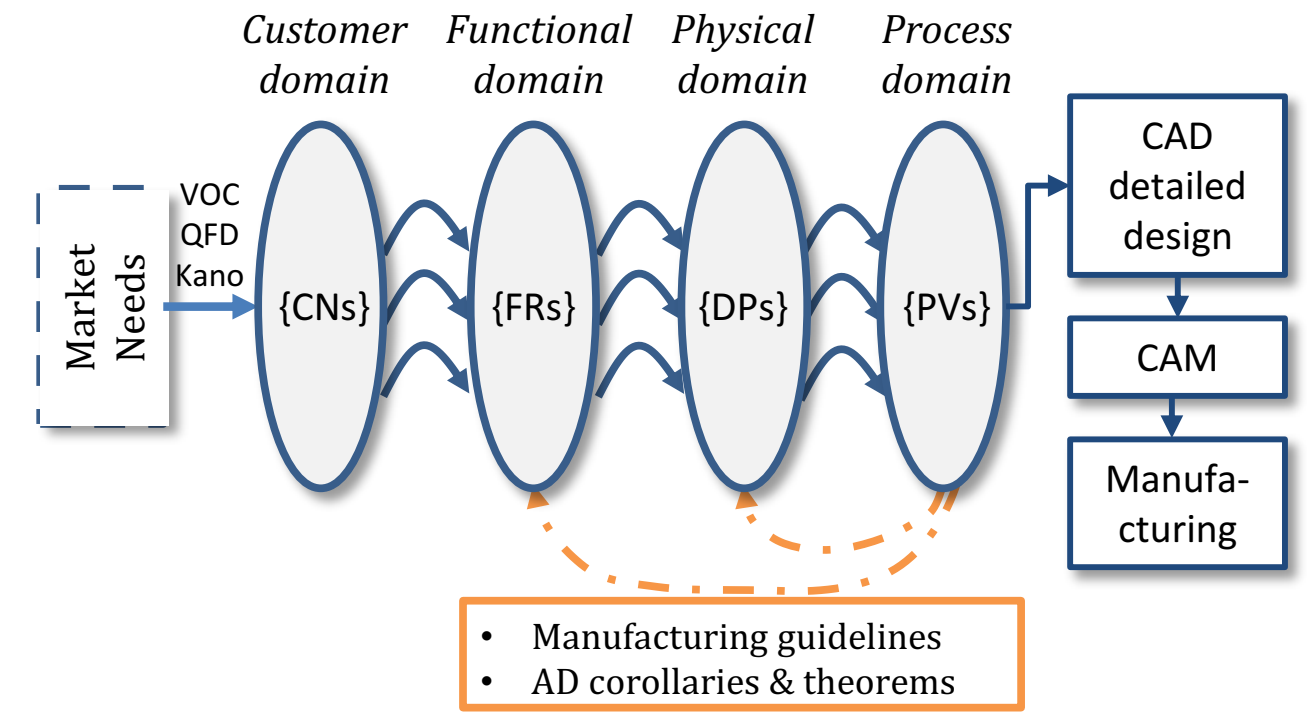

Figure 2. Axiomatic design framework tailored for the additive manufacturing

Therefore, in order for the manufacturing capabilities to be taken into consideration, the zigzag decomposition should not take place only between two adjacent domains (Figure 3), but through the three main domains (functional, physical and process) as can be seen in Figure 4. Such wider decomposition can be assisted by guidelines for manufacturing that can be obtained by the practitioners and the literature review. Additionally, simulation and 
process modelling can assist in the decision of the process variables as will be shown in the case study.

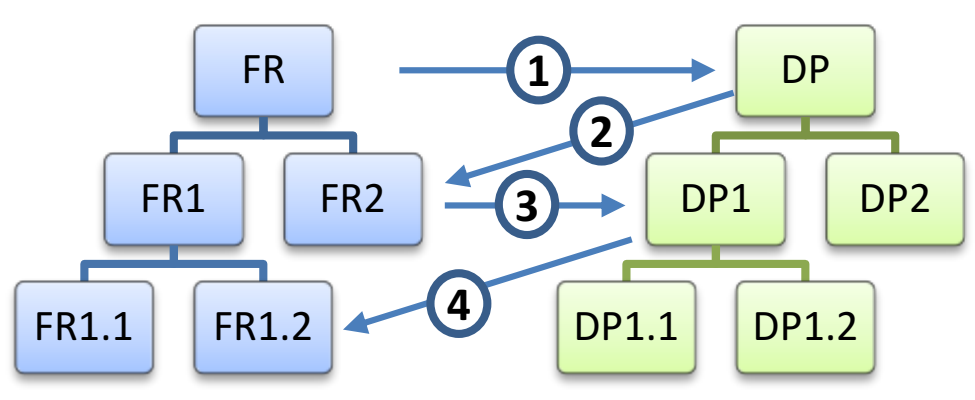

Figure 3. Traditional axiomatic design mapping between two adjacent domains.

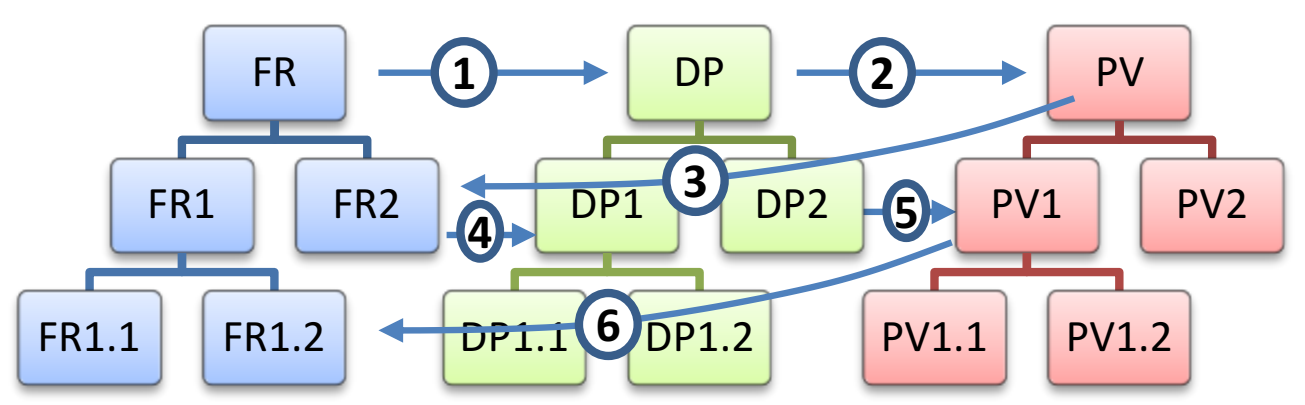

Figure 4. Axiomatic design mapping for considering manufacturing process capabilities during the early design phases.

Improving the manufacturability of the design from such an early stage allows the direct linking of the axiomatic design with the CAD software and subsequently the CAM tool for the planning of the manufacturing, as can be seen in Figure 2.

\subsection{Additive Manufacturing Guidelines}

As mentioned, the current practice with regards the assessment of the manufacturability of a component takes place after the design phase has been almost finalized. In order however, for these constraints to be considered early in the design process, even at the conceptual phase of the design, a set of rules or guidelines are needed. In order to collect such design guidelines, further to the thorough literature review, questionnaires were used to capture the practitioners' views. The literature review was performed in order to identify such guidelines from academic papers and simultaneously additive manufacturing OEMs 
were contacted (either by direct personal communication, or through the available information in their websites). However, since the goal was to better understand how these constraints are "interpreted" by the end users, the questionnaire was developed for rapid manufacturing bureaus based on the initial literature review and internet findings in order to assess the use by them. The constraints identified were ranked by the respondents and examples were requested for each of these constraints.

35 rapid manufacturing bureaus with expertise in both metallic and plastic additive manufacturing technology were contacted within the UK, with 22 responds received in a period of three months. The constraints that were collected are applicable to most of the additive manufacturing techniques, and can be grouped into the following design guidelines and limitations:

- Avoidance of enclosed hollow volumes

- Selection of proper clearances.

- Minimum feature size.

- Consideration of surface finish.

- Selection of materials and resulting mechanical properties.

- Consideration of the maximum working volume.

- Building time and cost.

The first three guidelines are specified during the design phase of the component, whereas the remaining ones are function of the specific technology used and the process parameters selection and decisions. Indicatively, enclosed hollow volumes might be desirable for reducing the weight of a component, but in general they will be filled with support material that is difficult to remove after the finishing process. Such problems can be addressed in the design phase by including gates to such areas. With regards the clearances, the standard achievable tolerances for most of the additive manufacturing machines are in the range of \pm $0.005^{\prime \prime}[15]$. The surface finish of additive manufactured parts can be controlled through the proper selection of process parameters, part orientation and material selection.

Furthermore, as indicated by Klahn et al. [16], additive manufacturing cannot be considered to replace conventional manufacturing processes, but should be considered when the 
design goal is to develop products that present either of the following criteria: integrated design, individualization, lightweight design and efficiency.

\section{Case Study}

For the validation of the proposed framework, a bracket that traditionally is manufactured through milling of an aluminium alloy 6082-T6 block (Figure 5), was selected as a case study. The bracket is composed of three small recesses on the top surface that are used to position and secure the bracket using three screws. The bracket needs to operate using existing clamping components. It must be also compatible with the interfaces of the existing mounting rail in the bottom of the bracket. The bracket is subject to three orthogonal, nonconcurrent shock loads. The final part needs to be as light as possible and should be easily cleaned.

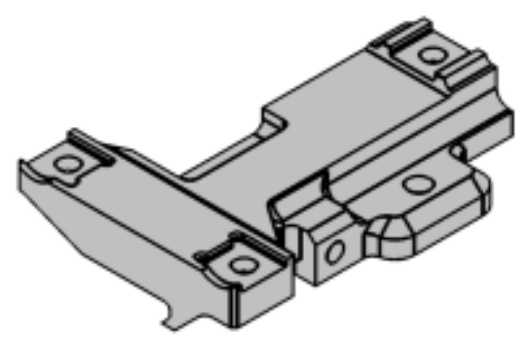

Figure 5. Case study design for manufacturing using machining

\subsection{Decomposition}

In adopting the axiomatic design methodology, the first step is to define the Functional Requirements (FRs) based on the customer needs. The highest-level functional requirement, which serves as the mission statement is shown as FRO in Table 1. The previous paragraph can be considered as the design brief, and thus the design parameter (DP) that will satisfy the functional requirement is considered to be surface topology optimization and can be denoted as DPO. The design that will result from such a design parameter can be manufactured using additive manufacturing and thus this is considered to be the highest level process variable (PV0). Few more decompositions will lead to the DPs and PVs with more specific details, as can be shown in Table 1 . The corresponding DPs and PVs are derived following the extended zigzagging method proposed in the previous section. 
Table 1. High level functional requirements, design parameters and process variables and first level decomposition

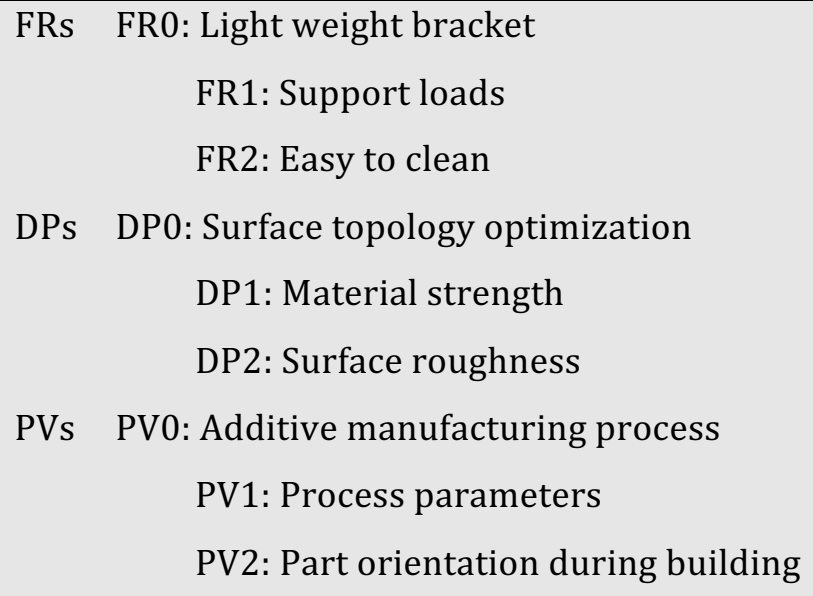

DPO has been identified as surface topology optimization. Since the functional requirement is a lightweight bracket, the structure of the bracket needs to be optimized. Topology optimisation is a systematic method, based on finite element analysis, to produce a strong part with minimum use of material, exhibiting an organic looking structure. Stress distribution and deformations are calculated trough finite element simulation of the existing model, in order to decide where material is redundant. The initial geometry finite element analysis and the resulted organic shape of the bracket are shown in Figure 6 . The only technology that can replicate with detail such organic structures is the additive manufacturing.

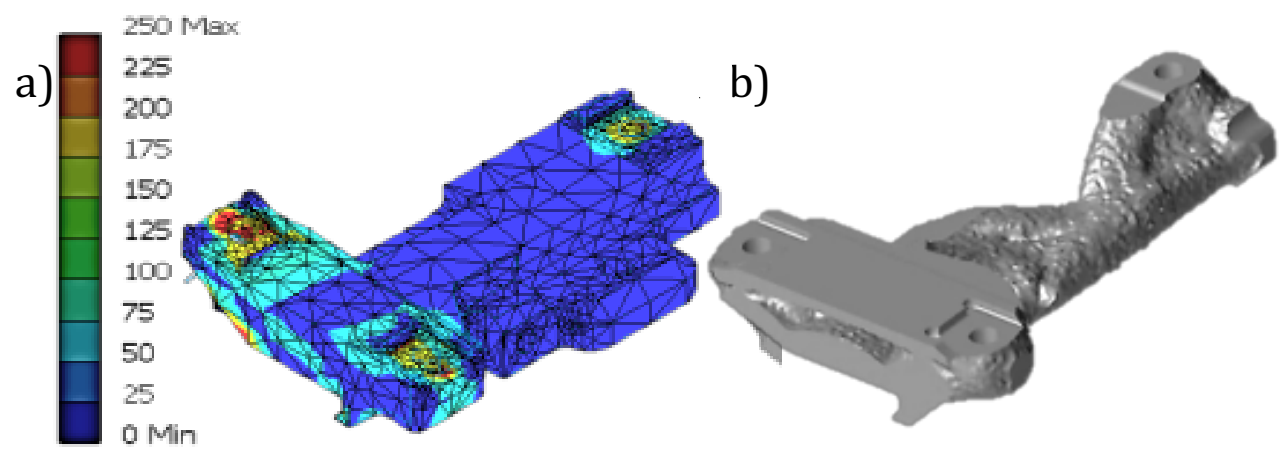

Figure 6. (a) FEA applied to the original bracket design and (b) Optimised shape using topology optimisation 
The decomposition to lower levels is achieved using the zigzagging method. For example the second level of FRs have been identified as the requirement for the bracket to support the operating loads (FR1) and the requirement for the component to be easily cleaned (FR2). The design parameters that can achieve such requirements, keeping in mind the manufacturing guidelines, were considered to be the strength of the material (DP1) and the surface roughness (DP2). By proper selection of the process parameters (PV1) both strength and surface roughness of the component can be controlled. Additionally, the orientation of the component (PV2) during "building" will affect the surface quality. The functional requirements, the design parameters and the process parameters can be further decomposed; indicatively DP1 could be decomposed into DP1.1 "static loads" and DP1.2 "dynamic loads". However, usually the decomposition is terminated, when a level is reached where the FRs can be fully satisfied by the selected set of DPs, and subsequently such DPs can be fully controlled by the selected PVs. The integrated product and process decomposition diagram can help in visualizing the zigzagging process [17], and is presented in Figure 7.

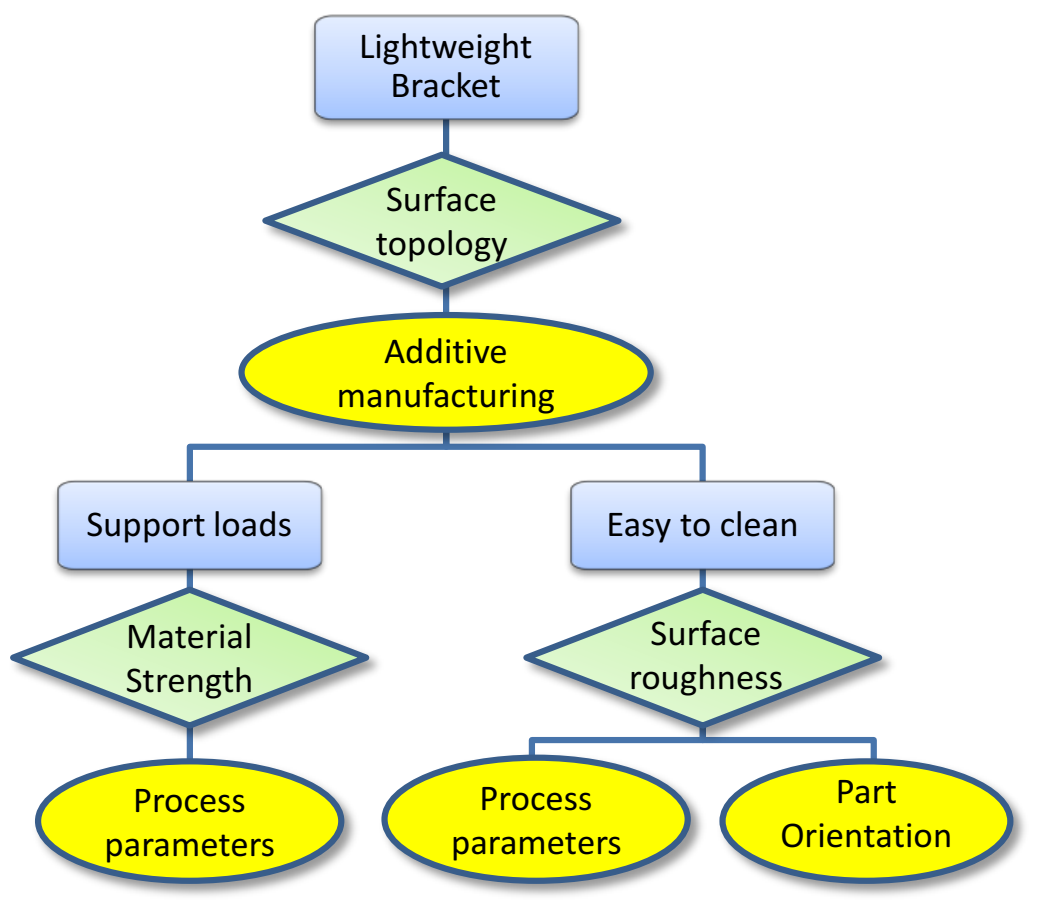

Figure 7. Integrated product and process decomposition (rectangles denote FRs, lozenges DPs and ellipses PVs) 


\subsection{Independence axiom}

Suh [11], [12] has presented the mathematical background of the axiomatic design. The mapping of the functional requirements to the design parameters (through the hierarchical decomposition and the zigzagging) is described by the following equation:

$\{F R s\}=[A]\{D P s\}$

where $\{F R s\}$ and $\{D P s\}$ are the functional requirements and design parameters vectors respectively and $[A]$ is the design matrix. Similarly the mapping between the physical and the process domains is denoted by:

$$
\{D P s\}=[B]\{P V s\}
$$

where $\{\mathrm{PV}\}$ is the process variables vector and $[\mathrm{B}]$ the matrix linking the physical and the process domain. Following the wider decomposition proposed in Figure 4, eqs. (1) and (2) can be combined into the following equation:

$\{F R s\}=[C]\{P V s\}$

where $[\mathrm{C}]=[\mathrm{A}] \times[\mathrm{B}]$ is the matrix linking the requirements to the process variables.

The independence axiom is assessed by the shape and the content of the matrix. As per Suh's notation, when the matrix is diagonal then the design is considered to be "uncoupled", when triangular then it is classified as "decoupled", otherwise it is characterized as "coupled". An uncoupled design is the ideal whereas the decoupled design is also acceptable when the DPs (and subsequently the PVs) are selected in the correct order. Therefore, in the present approach all three matrices $([A],[B]$ and $[C])$ need to be checked, and the various vectors must be optimized in order to achieve at least decoupled solutions. For the proposed solution the three matrices are presented in the following equations, with $\mathrm{X}$ indicating strong influence whereas 0 indicates weak influence between the FR and DP:

$\left\{\begin{array}{l}F R 1 \\ F R 2\end{array}\right\}=\left[\begin{array}{ll}X & 0 \\ 0 & X\end{array}\right]\left\{\begin{array}{l}D P 1 \\ D P 2\end{array}\right\}$

$\left\{\begin{array}{l}D P 1 \\ D P 2\end{array}\right\}=\left[\begin{array}{ll}X & 0 \\ X & X\end{array}\right]\left\{\begin{array}{l}P V 1 \\ P V 2\end{array}\right\}$ 


$$
\left\{\begin{array}{l}
F R 1 \\
F R 2
\end{array}\right\}=\left[\begin{array}{ll}
X & 0 \\
X & X
\end{array}\right]\left\{\begin{array}{l}
P V 1 \\
P V 2
\end{array}\right\}
$$

Eqs. (4) to (6) indicate that the design is acceptable from the independence axiom point of view, as design matrix $[A]$ is diagonal, whereas matrices $[B]$ and $[C]$ are triangular. Figure 7 is also conveying the same message. Thus the result of this analysis indicates that the initial decomposition proposed is feasible, and the designs that adhere to such decomposition are acceptable. A number of concept designs can thus be developed and proposed, as shown in Figure 8.

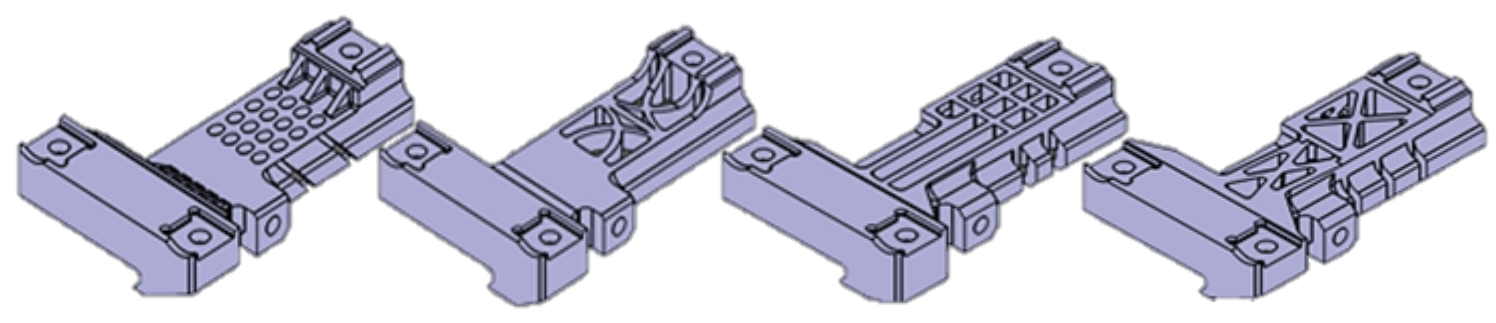

Figure 8. Alternative concepts that comply with the first axiom

\subsection{Information axiom: comparison of different solutions}

The comparison of the different acceptable solutions that conform to the independence axiom is performed based on the information axiom. The information axiom was defined by Suh [12] with regards the information content needed for satisfying a given functional requirement. For each functional requirement the information content can be calculated as:

$I_{i}=\log _{2}\left(\frac{1}{p_{i}}\right)$

where $p_{i}$ is the probability for achieving the functional requirement $F R_{i}$. In literature, the probability is given in terms of design range (the tolerances that the designer wishes his design to meet) and the system range (what the system is capable of delivering). In the last few years a number of approaches have been presented where the information is expressed in fuzzy logic terms in order to account for qualitative information [13].

For the case discussed in the present study, the information content needs to be defined for the two functional requirements. Since the support load is specified through the design 
specifications, all the alternative designs will be able to support this. Furthermore, we can safely assume that all concepts will meet FR1 (support load), as the same material will be used with the same technology (thus the influencing process variables do not affect this functional requirement). Thus only the "ease to of cleaning" will be considered here. High values of surface roughness can result in accumulation of dust and dirt, thus surface roughness needs to be minimized with a maximum allowable value. Process models can be employed for this reason to quantitatively describe the capability of the process.
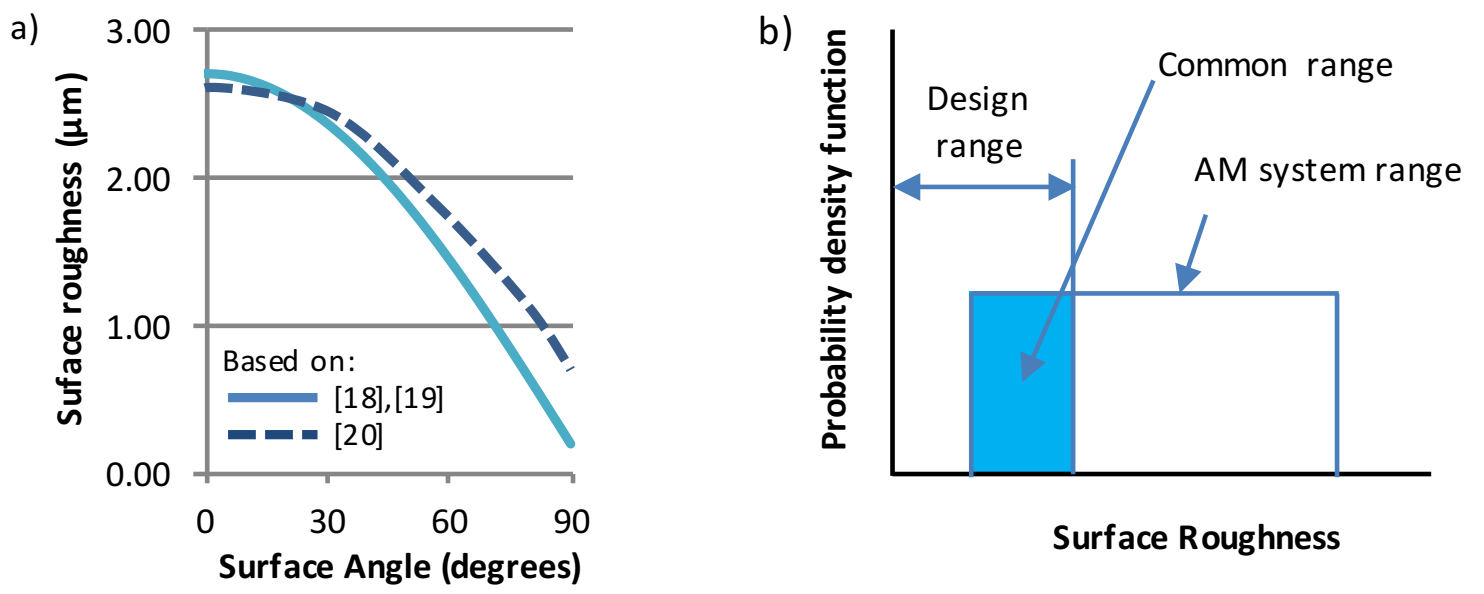

Figure 9. (a) Surface roughness as a function of surface angle and (b) the system and design range and the common range

Figure 9.a presents the results of such models connecting the angle of inclination with the resulting surface roughness for the case of stereolithography. The average surface roughness of SLA-produced parts was estimated as a function of the layer thickness and the angle of the inclined surface [18], [19]. Modelling was based on simplistic trigonometry assumptions, while the surface roughness $\left(R_{a}\right)$ could be calculated according to the following equation:

$$
R_{a}=D_{p} \cdot \ln \left[\sqrt{\frac{2}{\pi W_{0} V_{S} E_{C}}} \frac{P_{L}}{4 \tan \theta}\right]-O C \frac{\sin \theta}{4 \tan \theta}
$$

Where $D_{p}$ is the depth of penetration, $P_{L}$ is the nominal laser power, $W_{0}$ is the laser beam spot diameter, $V_{S}$ is the laser scanning speed, $E_{C}$ is the critical exposure time, $O C$ is the overcure and $\vartheta$ is the inclination angle. 
An analytical model presented from Reeves and Cobb in [20] for SLA surface roughness that took into consideration the layer profile as well whether the plane was up-facing or downfacing was also used in the present study and presented in figure 9.a. Similar trends can be observed between the two models.

Similar models can be derived for other additive processes as well (indicatively fused deposition modelling has been modelled in [21] and [22], Selective Laser Melting in [23], 3D printing in [81], Laminated object manufacturing in [33], etc.).

Considering uniform probability functions, as shown in Figure 9.b, the information content of each approach can be related to the amount of inclined features in the selected design, with the designs having more inclined surfaces to present higher information content. The design that exhibited the minimum information content was selected (Figure 10).

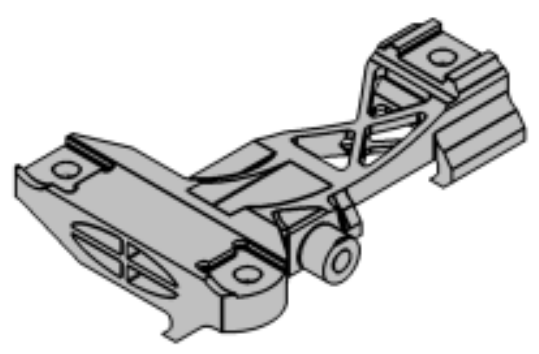

Figure 10. Case study design for manufacturing using AM

\section{Conclusions}

Design for additive manufacturing is limited by the use of methods and approaches developed for conventional manufacturing. In the present work, the axiomatic design theory was adapted and zigzagging decomposition was expanded to take into consideration the manufacturing limitations and capabilities from the early phases of design. For this reason manufacturing guidelines and constraints were captured from additive manufacturing practitioners. The method was validated for the case of additive manufacturing of a component. The axiomatic design was combined with surface topology optimization for the high level decomposition and was presented in the present paper for the first time. Based on such a combined approach, designers can take advantage of the processes capabilities in order to design complex designs by using unexplored regions of the design space and assess their creativity using the two axiomatic design theorems. 


\section{References}

[1] Kruth J-P, Leu MC, Nakagawa T (1998) Progress in Additive manufacturing and rapid prototyping. CIRP Annals - Manufacturing technology 47(2): 525-540

[2] Wohlers (2013) Wohlers Report: Additive Manufacturing and 3D printing state of the industry. Wohlers Associates

[3] RAEng (2013) Additive Manufacturing: opportunities and constraints. Royal Accademy of Engineering

[4] Levy GN, Schindel R, Kruth JP (2003) Rapid Manufacturing and rapid tooling with layer manufacturing (LM) technologies, state of the art and future perspectives. CIRP Annals - Manufacturing Technology 52(2): 589-609.

[5] Rodrigue H, Rivette M (2010) An assembly-level design for additive manufacturing methodology. Proceedings of IDMME - Virtual concept 2010, P20

[6] Vayre B, Vignat F, Villeneuve F (2012). Design for additive manufacturing. Procedia CIRP 3:632-637.

[7] Podshivalov L, Gomes CM, Zocca A, Guenster J, Bar-Yoseph C, Fisher A (2013) Design, analysis and additive manufacturing of porous structures for biocompatible microscale scaffolds. Procedia CIRP 5:247-252.

[8] Ponche R, Kerbrat O, Mognol P, Hascoet J-Y (2014). A novel methodology of design for additive manufacturing applied to additive laser manufacturing process. Robotics and Computer-Integrated Manufacturing 30:389-398.

[9] Adam GAO, Zimmer D (2014). Design for additive manufacturing - Element transitions and aggregated structured. CIRP Journal of Manufacturing Science and Technology 7:20-28.

[10] Salonitis K, Al Zarban S (2015). Redesign Optimization for Manufacturing Using Additive Layer Techniques. Procedia CIRP 36:193-198

[11] Suh NP (1990) The Principles of Design, Oxford University Press, New York

[12] Suh NP (2001) Axiomatic Design - Advances and Applications, Oxford University Press

[13] Kulak O, Cebi S, Kahraman C (2010) Applications of axiomatic design principles: A literature review. Expert Systems with Applications 37:6705-6717

[14] Suh, NP (1988) Basic Concepts in Design for Producibility. CIRP Annals - Manufacturing Technology 37/2:559-567 
[15] Gibson I, Rosen D, Stucker B (2010) Additive manufacturing technologies. New York: Springer.

[16] Klahn C, Leutenecker B, Meboldt M (2014) Design for Additive Manufacturing Supporting the substitution of components in series products. Procedia CIRP 21:138143

[17] Concalves-Coehlo AM, Mourao AJF (2007) Axiomatic design as support for decisionmaking in a design for manufacturing context: A case study. International Journal of Production Economics 109:81-89

[18] Salonitis K (2014) Stereolithography. In: Comprehensive Materials Processing (Masood, S., Ed.); Elsevier Ltd., Vol. 10, pp 19-67.

[19] Salonitis K, Tsoukantas G, Stavropoulos P, Stournaras A (2003) A critical review of stereolithography process modeling, In: Virtual Modelling and Rapid Manufacturing Advanced Research in Virtual and Rapid Prototyping, pp 377-384

[20] Reeves PE, Cobb RC (1997) Reducing the surface deviation of stereolithography using in-process techniques. Rapid Prototyping Journal 3(1):20-31

[21] Kaji F, Barari A (2015) Evaluation of the Surface Roughness of Additive Manufacturing Parts Based on the Modelling of Cusp Geometry. IFAC-PapersOnLine 48-3 (2015) 658663

[22] Anitha R, Arunachalam S, Radhakrishan P (2001) Critical parameters influencing the quality if prototypes in fused deposition modeling. Journal of Materials Processing Technology 118(1-3):385-388

[23] Strano G, Hao L, Everson RM, Evans KE (2013) Surface roughness analysis, modelling and prediction in selective laser melting. Journal of Materials Processing Technology 213(4):589-597

[24] Jee HJ, Sachs E (2000) A visual simulation technique for 3D printing. Advanced Engineering Software 31:97-106

[25] Chryssolouris G, Kechagias JD, Kotselis JL, Mourtzis DA, Zannis SG Surface roughness modelling of the Helisys laminated object manufacturing (LOM) Process. 8th European Conference on Rapid Prototyping and Manufacturing, Nottingham, pp 141-152 
2016-03-03

\section{Design for additive manufacturing based on the axiomatic design method}

Salonitis, Konstantinos

Springer

Salonitis K, Design for Additive Manufacturing sased on Axiomatic Design Method, The International Journal of Advanced Manufacturing Technology, October 2016, Volume 87, Issue pÿ1, pp 989996

http://dx.doi.org/10.1007/s00170-016-8540-5

Downloaded from Cranfield Library Services E-Repository 